\title{
Infections and Use of Antibiotics in Patients Admitted for Severe Acute Pancreatitis: Data from the EPIC II Study
}

\author{
Jan J. De Waele, Jordi Rello, ${ }^{1}$ Antonio Anzueto, ${ }^{3}$ Rui Moreno,, Jeffrey Lipman,, Yasser Sakr, \\ Peter Pickkers, ${ }^{7}$ Marc Leone, ${ }^{8}$ Andrew Ferguson, ${ }^{9}$ Lavi Oud, ${ }^{10}$ \\ and Jean-Louis Vincent, ${ }^{11}$ the EPIC II Investigators
}

\begin{abstract}
Background: Infectious complications are frequent in severe acute pancreatitis (SAP) but multinational epidemiologic data are lacking. The aim of the study was to analyze the characteristics of the infectious complications and antimicrobial use in this setting.

Methods: One-day point prevalence study of infection in critically ill patients (Extended Prevalence of Infection in the ICU-II study), performed in 1,265 ICUs in 75 countries.

Results: Of the 13,796 patients in the study, 159 were admitted with SAP. One-hundred sixteen (73\%) had infections: $31 \%$ intra-abdominal, $16 \%$ extra-abdominal, and $26 \%$ both. Gram-negative bacteria were more prevalent than gram-positive organisms, anaerobes, or fungi. Therapeutically, penicillins and other beta-lactams were used most frequently. Prophylactic antibiotics were administered to $24 \%$ of the patients with SAP.

Conclusions: Infections are frequent in patients admitted with SAP; most are intra-abdominal infections. Microbiology is diverse with gram-negative micro-organisms most frequently isolated. Most patients admitted to the ICU for SAP receive antibiotics at some point.
\end{abstract}

Severe acute pancreatitis (SAP) is often complicated by pancreatic and extra-pancreatic infections [1,2]. Although infection of pancreatic necrosis is an important source of morbidity and mortality [3], other infections, such as pneumonia and bacteremia, are frequent and may impact outcomes [1]. Early diagnosis and prompt treatment with antibiotics and appropriately-timed source control interventions are the mainstays of therapy of infected necrosis [4,5]. Use of prophylactic antibiotics to prevent infection was advocated in the past, based largely on small, non-blinded studies. However, recent randomized controlled trials could not demonstrate any advantage of this tactic in patients with SAP [6,7]. But despite recommendations against the routine use of prophylaxis in this setting [8], many clinicians continue to administer antibiotics to patients without culture-proved infection [9-11].

Antibiotics are best reserved for the treatment of established infection [5], which can be diagnosed reliably using fine-needle aspiration, guided either by computed tomography or ultrasound [12]. However, data regarding current antibiotic use in patients with SAP admitted either to the intensive care unit (ICU) or to the regular ward are limited. The Extended Prevalence of Infection in the ICU II (EPIC II) was a large point-prevalence study of infections in the ICU. The study showed that one-half of all ICU patients on the

\footnotetext{
${ }^{1}$ Department of Critical Care Medicine, Ghent Medical School and University Hospital, Ghent, Belgium.

${ }^{2}$ Critical Care Department, Hospital Vall d'Hebron, CIBERES, Universitat Autonoma de Barcelona, Spain.

${ }^{3}$ University of Texas Health Science Departments of Pulmonary/Critical Care, and Internal Medicine, and South Texas Veterans Health Care System, San Antonio, Texas.

${ }^{4}$ UCINC, Hospital de São José, Centro Hospitalar de Lisboa Central, Lisbon, Portugal.

${ }^{5}$ Discipline of Anesthesiology and Critical Care, The University of Queensland School of Medicine, and Department of Intensive Care Medicine, Royal Brisbane and Women's Hospital, Herston QLD, Australia.

${ }^{6}$ Department of Anesthesiology and Intensive Care, Friedrich-Schiller-University, Jena, Germany.

${ }^{7}$ Department of Intensive Care, Radboud University Nijmegen Medical Centre, Nijmegen, Netherlands.

${ }^{8}$ Department of Intensive Care and Anesthesiology, Hôpital Nord, AP-HM, Unité de Recherche en Maladies Infectieuses et Transmissibles (URMITE), Aix-Marseille University, Marseilles, France.

${ }_{9}^{9}$ Department of Intensive Care Medicine, Craigavon Area Hospital, Portadown, United Kingdom.

${ }^{10}$ Division of Pulmonary and Critical Care Medicine, Texas Tech University HSC, Odessa, Texas.

${ }^{11}$ Department of Intensive Care, Erasme Hospital, Université Libre de Bruxelles, Brussels, Belgium.
} 
study day were infected and that $71 \%$ were treated with antibiotics [13]. Intra-abdominal infections ranked second and were present in $19.6 \%$ of the patients. The EPIC II study database offers a unique opportunity to study the use of antibiotics, either as prophylaxis or as therapy, in SAP patients from multiple centers around the world. The objective of this study was thus to analyze the characteristics of the infectious complications and the use of antibiotics in EPIC II study patients admitted to the ICU with SAP.

\section{Patients and Methods}

The EPIC II one-day point prevalence study of infection in critically ill patients was performed on May 8, 2007. Demographic, physiologic, bacteriologic, and therapeutic data were collected from 13,796 adult (age $>18 \mathrm{y}$ ) patients in 1,265 participating ICUs from 75 countries (see Appendix online at www.liebertpub.com/sur) on the study day, as described previously [13]. Local ethical committee approval at each participating center was expedited or waived due to the purely observational nature of the study. Infection was defined according to the criteria of the International Sepsis Forum and classified by the attending physician. Microbiologic analyses were performed locally. Participating ICUs were asked to provide patient follow-up until hospital discharge or for $60 \mathrm{~d}$ (until July 9, 2007), and ICU and hospital outcomes were recorded. For the purposes of this study, we selected the patients who were admitted to the ICU because of SAP.

\section{Statistical analysis}

Statistical analyses were performed using PASW Statistics 18 for Windows (SPSS Inc, Chicago, USA). Data are presented as mean ( \pm standard deviation), or percentage as appropriate, except for length of stay, which is reported as median (interquartile range [IQR]). Difference testing between groups was performed using a Mann-Whitney test or Fisher exact test as appropriate. All tests were two-tailed, and a $\mathrm{p}$ value $<0.05$ was considered statistically significant.

\section{Results}

\section{Patient characteristics}

One-hundred fifty-nine of the 13,796 patients (5\%) were admitted to the ICU because of SAP; $65 \%$ were male and the mean age was $57 \pm 15$ years. The mean Simplified Acute Physiology Score II was $34 \pm 14.2$. Patients with SAP stayed in the ICU for a median of 25 (10-53) days; ICU and hospital mortality rates were $23 \%$ and $27 \%$, respectively. The distribution of patients according to their length of stay prior to the study day is shown in Figure 1.

Patients with SAP were admitted to ICUs across all continents, with the highest proportion coming from Western Europe (54\%), followed by Latin America (19\%), Eastern Europe and Russia (9\%), Asia (7), North America (6\%), the Pacific region (3\%), and Africa (1\%).

\section{Infections}

One hundred sixteen of the 159 patients with SAP (73\%) were diagnosed with an infection; $50(31 \%)$ patients had intraabdominal infections, 25 (16\%) had extra-abdominal infections,

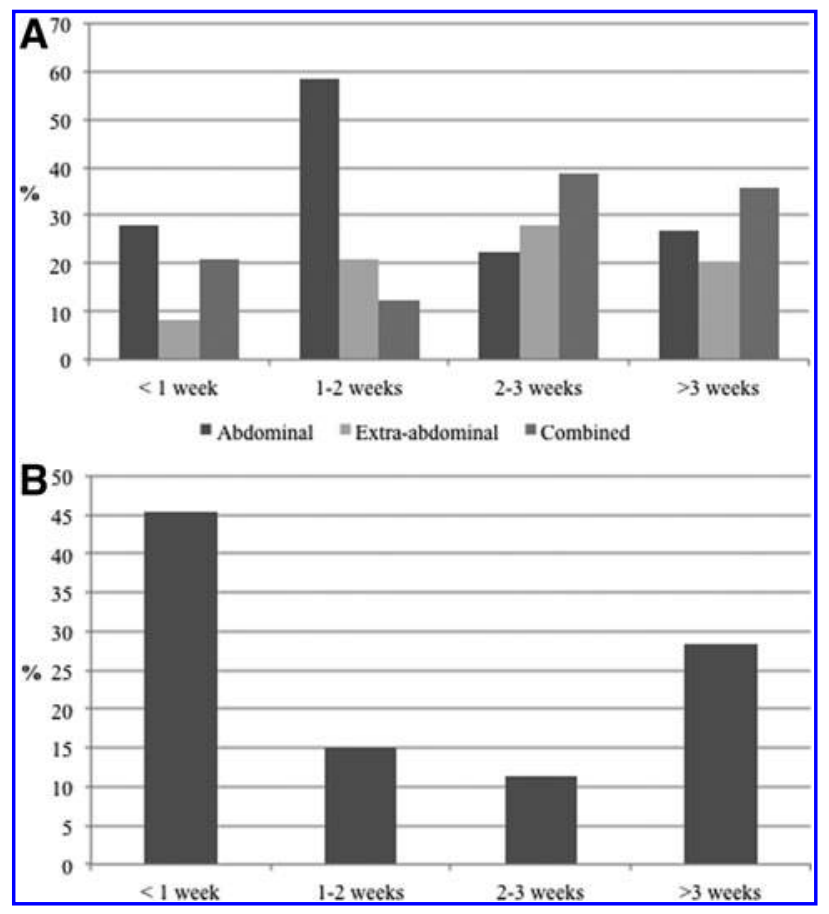

FIG. 1. (A) Distribution of patients with severe acute pancreatitis and (B) infection rate according to length of stay in the intensive care unit prior to the study day.

and $41(26 \%)$ had a combination of the two (Fig. 2). Extraabdominal infections included respiratory tract infections in 44 patients (28\%), blood stream infections in 22 patients (14\%), both catheter-related infections in six (4\%), genito-urinary tract infections in six patients (4\%), skin infections in five (3\%), renal infections in two patients $(1 \%)$ and other infections in four patients (3\%).

In SAP patients with intra-abdominal infections, 66 different micro-organisms were isolated, with gram-negative bacteria $(\mathrm{n}=29)$ being the most prevalent (see Table 1); $32 \%$ of the infections were polymicrobial. Escherichia coli and Pseudomonas species were the most frequently isolated (in 10 patients each) bacteria. Candida albicans was detected in eight patients.

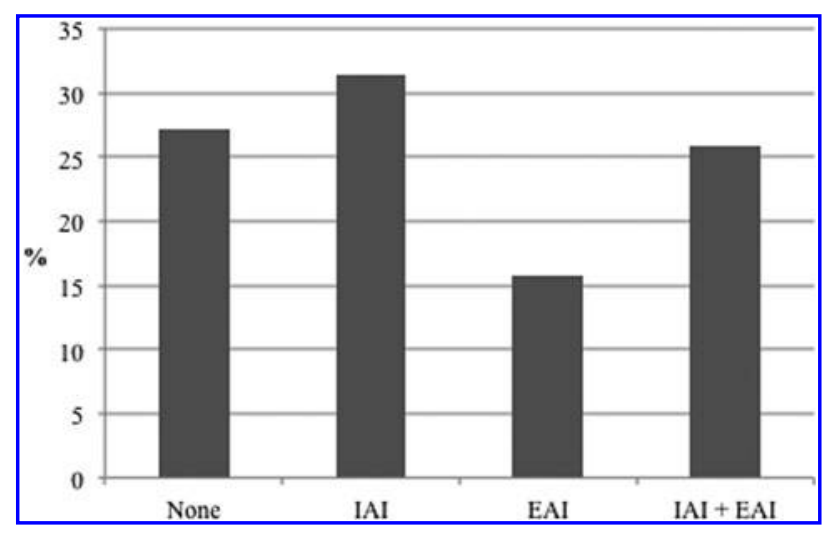

FIG. 2. Distribution of sites of infection in patients admitted with severe acute pancreatitis. IAI = intra-abdominal infection; EAI = extra-abdominal infection. 
Table 1. Microbiology of Intra-AbDominal Infections in Patients With SeVERE ACUte Pancreatitis

\begin{tabular}{lr}
\hline Gram-positive microorganisms $(\mathbf{n}=\mathbf{2 5})$ & 1 \\
MSSA & 2 \\
MRSA & 3 \\
MSSE & 2 \\
MRSE & 9 \\
Streptococcus group D - MS & 6 \\
Streptococcus group D - MR & 1 \\
Streptococcus group A/B/C & 1 \\
Streptococcus (other) & 10 \\
Gram-negative micro organisms (n=29) & 3 \\
Escherichia coli & 6 \\
Enterobacter species & 3 \\
Klebsiella species & 1 \\
Proteus species & 1 \\
Salmonella species & 1 \\
Pseudomonas species & 1 \\
Stenotrophomonas maltophilia & 1 \\
Acinetobacter species & 0 \\
Other & \\
ESBL-producing gram-negative organisms & 3 \\
Anaerobes (n=3) & \\
Clostridium species & 8 \\
Fungi (n=9) & 1 \\
Candida albicans & \\
Candida non-albicans & \\
Other $(\mathbf{n}=\mathbf{1})$ & \\
\hline
\end{tabular}

MSSA = methillicin-sensitive Staphylococcus aureus; MRSA = methicillin-resistant Staphylococcus aureus; $\mathrm{MSSE}=$ Staphylococcus epidermidis; MRSE = methicillin-resistant Staphylococcus epidermidis; $\mathrm{MS}=$ methillicin-sensitive; $\quad \mathrm{MR}=$ methicillin-resistant; $\quad \mathrm{ESBL}=$ extended-spectrum beta-lactamase.

The ICU length of stay was significantly longer in infected versus non-infected SAP patients (median, 29 [11-63] days vs. 14 [6-30]; $\mathrm{p}<0.001)$. Mortality rates in infected patients also were higher in the ICU than in non-infected patients (ICU mortality, $28 \%$ vs. $10 \%$; $\mathrm{p}=0.017$; hospital mortality. $31 \%$ vs. $17 \%, \mathrm{p}=0.084)$.

\section{Antimicrobial use}

A total of 133 of the 159 patients (84\%) received antibiotics. Penicillins and other beta-lactams were the antibiotics used most frequently to treat established infections (Table 2). Glycopeptides and anti-fungal agents were also used regularly.

Table 2. Antibiotics Administered for Established INFECTIONS IN 116 PATIENTS ADMITTED For SeVERe Acute PANCREatitis

Carbapenem

Anti-fungal

Glycopeptide

Penicillin

Fluoroquinolone

Cephalosporin

Aminoglycoside

Other

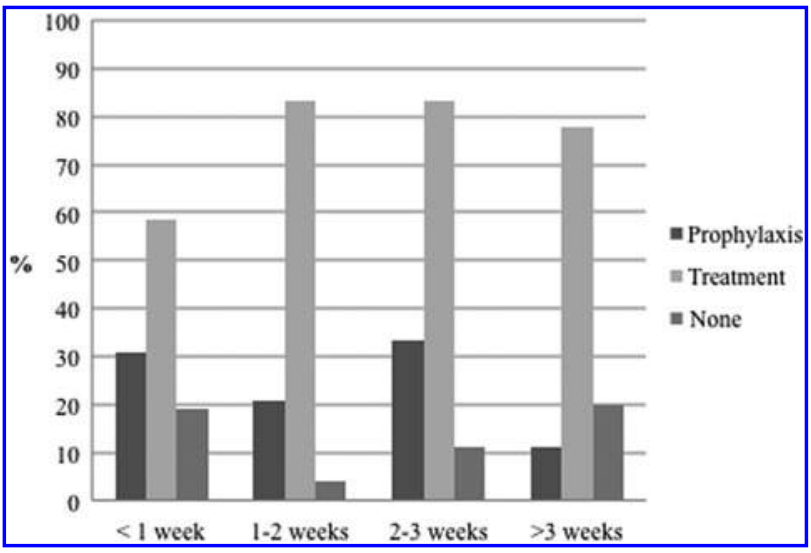

FIG. 3. Proportion of patients receiving prophylactic or therapeutic antibiotics on the study day according to the length of stay in the intensive care unit prior to the study day.

Prophylactic antibiotics were administered to 38 of the 159 patients (24\%), most often in patients who had been present on the ICU for three weeks or less (Fig. 3); in this latter subgroup of 72 patients, $22(31 \%)$ were receiving prophylactic antibiotics (most frequently beta-lactams; Table 3). Twelve patients $(8 \%)$ received a prophylactic anti-fungal agent.

Among the antifungals administered for prophylaxis or therapy, fluconazole was used most frequently: 34 patients received fluconazole, six some formulation of amphotericin $\mathrm{B}$, four caspofungin, four voriconazole, and one patient received an unspecified anti-fungal agent.

\section{Discussion}

In this cohort of patients admitted to the ICU with SAP, infections were frequent and mortality was high. Although intra-abdominal infections were most prevalent (occurring in more than one-half the patients), extra-abdominal infections also were common, with the lung being the most frequent source of extra-abdominal infection. Most patients with SAP were receiving antibiotics on the study day. Gram-negative organisms were isolated most frequently from patients with intra-abdominal infections, whereas yeasts were isolated less frequently in these patients.

The high incidence of infectious complications is not surprising because infection is one of the major complications in SAP and is strongly correlated with organ dysfunction. In a meta-analysis of 14 studies by Petrov et al. [3], 1,478 patients had acute pancreatitis; $314(21 \%)$ of them

Table 3. Prophylactic Antibiotics administered to Patients with Severe Acute Pancreatitis

$\begin{array}{ll}\text { Carbapenem } & 17\end{array}$

Antifungal 12

Aminoglycoside $\quad 5$

Cephalosporin 4

Fluoroquinolone 3

Penicillin $\quad 3$

Other AB 11

$\mathrm{AB}=$ antibiotics. 
developed infected pancreatic necrosis, with an incidence in the individual reports ranging from $4 \%$ to $63 \%$. In the group of 713 patients considered for the PROPATRIA study, of whom only 154 had documented pancreatic necrosis, Besselink et al. reported infected pancreatic necrosis in 98 patients [1], yielding an incidence of $63.3 \%$. In a multi-center audit from Italy, 56 of 161 patients with demonstrated pancreatic necrosis developed infection [10]. It was not clear how many patients were admitted to the ICU, so it is difficult to compare that study to the present analysis.

The denominator is important when comparing the incidence of infection in different studies. Most studies in patients with SAP have included patients admitted to the ICU, as well as to general wards or high-dependency units, so differences in patient populations are likely to explain the observed variability in the incidence of infections in these patients. From the Simplified Acute Physiology Score II score, it can be assumed that the present study population was sicker, compared with that of most observational reports. Moreover, the design of the study (a point-prevalence study) may also affect the results. Patients with infected pancreatic necrosis have a protracted ICU course, which explains the long length of stay of the patients in this study, and increases the probability that infection of pancreatic necrosis was present. On the other hand, patients who have been admitted for one week or less (the largest group in the present study) are less likely to have developed infection; the median delay to infected pancreatic necrosis in the study by Besselink et al. was $26 \mathrm{~d}$ [1].

Although mortality was high overall, the separate effects of pancreatic or extra-pancreatic infections on outcome cannot be estimated from this study, as these data were not collected in the EPIC-II study. Petrov et al. reviewed the impact of infection and multiple organ dysfunction syndrome in patients with acute pancreatitis and reported that these two factors were equally important but when both were present, mortality rates were doubled [3]. Others have found increased mortality rates in patients with pneumonia or infected pancreatic necrosis and bacteremia [1].

As in other reports, gram-negative bacteria were most prevalent, with Escherichia coli the most frequently isolated microorganism. Notably, problematic organisms, such as Pseudomonas and Acinetobacter species, were common among the gram-negative organisms, but the same effect was not found among the gram-positive microorganisms. Remarkably, none of the reported microorganisms was extended-spectrum beta-lactamase producing.

Fungal infections are equally feared in this setting, yet fungi were less predominant in this series compared to previous reports $[14,15]$. The frequent use of anti-fungal agents in our study may explain these findings. Anti-fungals have been recommended often based on earlier studies that highlighted the importance of yeasts [16-18] and suggested an advantage of prophylactic administration of anti-fungal agents [19].

The use of antibiotics as prophylaxis was considerable in this study. Although the use of prophylactic antibiotics to prevent infected pancreatic necrosis was once adopted eagerly based on a number of small, uncontrolled studies [5], subsequent randomized controlled trials could not find any advantage of this strategy [6,7]. This observation was upheld in the most recent meta-analyses [20,21] and the approach is no longer recommended by scientific societies [8]. Our study shows that in clinical practice, these recommendations are often disregarded. A recent Italian survey similarly reported that antibiotic prophylaxis is widely used in clinical practice, with $25 \%$ of patients with mild or moderate SAP receiving antibiotic prophylaxis [10]. In addition, data from a large study on probiotics from The Netherlands (the PROPATRIA study) also showed that $20-25 \%$ of patients received antibiotics without proved infection [11]. However, patients in these studies were recruited before the most recent evidence was published, and therefore likely reflect the earlier treatment policy. The same comment may apply to the current analysis, although the 2004 Consensus Conference on the management of SAP already advised against the use of prophylactic antibiotics to prevent pancreatic infection [8]. The different classes of antibiotics used for prophylaxis reflect the heterogeneity of the initial studies on which antibiotic prophylaxis for acute pancreatitis patients was based. Therapeutic antibiotic choices also were diverse; the unpredictable microbiology and variable antibiotic susceptibility are probably the major explanations for this finding.

This study provides a real-life snapshot of infectious complications and antibiotic use in unselected patients admitted with SAP across different countries and continents. This cohort is unique but the study also has a number of limitations. As stated earlier, the population may be skewed towards more patients with prolonged lengths of stay and therefore may not accurately reflect the incidence of infection all SAP patients. The point prevalence nature of the study did not allow us to determine whether the prophylaxis was associated with different rates or patterns of infection. Furthermore, details regarding the etiology or severity of the pancreatitis were not available and microbiology was performed locally, with potential differences in methodology and reporting.

In conclusion, our results show that SAP is a relatively uncommon indication for ICU admission but is associated with high ICU mortality rates. Infections appear to be a major problem in this population of patients and, although intraabdominal infections are most frequent, a substantial proportion of patients are treated for infection at an extraabdominal site. Microbiology is diverse with gram-negative infections being most frequent, and fungal infections relatively uncommon. Most patients admitted to the ICU for SAP receive antibiotics. Antibiotic prophylaxis, although not supported by scientific evidence, is still practiced widely.

\section{Acknowledgments}

We thank Hassane Njimi, MSc, PhD, Department of Intensive Care, Ersme University Hospital, Brussels, Belgium, for his help with the data management and statistical analyses.

\section{Author Disclosure Statement}

No competing financial interests exist.

\section{References}

1. Besselink MG, van Santvoort HC, Boermeester MA, et al. Timing and impact of infections in acute pancreatitis. $\underline{\mathrm{Br} J}$ Surg 2009;96:267-273.

2. Charbonney E, Nathens AB. Severe acute pancreatitis: A review. Surg Infect (Larchmt) 2008;9:573-578. 
3. Petrov MS, Shanbhag S, Chakraborty M, et al. Organ failure and infection of pancreatic necrosis as determinants of mortality in patients with acute pancreatitis. Gastroenterology 2010;139:813-820.

4. van Santvoort HC, Besselink MG, Bakker OJ, et al. A stepup approach or open necrosectomy for necrotizing pancreatitis. N Engl J Med 2010;362:1491-1502.

5. DeWaele JJ. Use of antibiotics in severe acute pancreatitis. Expert Rev Anti Infect Ther 2010;8:317-324.

6. Dellinger EP, Tellado JM, Soto NE, et al. Early antibiotic treatment for severe acute necrotizing pancreatitis: A randomized, double-blind, placebo-controlled study. Ann Surg 2007;245:674-683.

7. Isenmann R, Runzi M, Kron M, et al. Prophylactic antibiotic treatment in patients with predicted severe acute pancreatitis: A placebo-controlled, double-blind trial. Gastroenterology 2004;126:997-1004.

8. Nathens AB, Curtis JR, Beale RJ, et al. Management of the critically ill patient with severe acute pancreatitis. Crit Care Med 2004;32:2524-2536.

9. Andersson B, Andren-Sandberg A, Nilsson J, Andersson R. Survey of the management of acute pancreatitis in surgical departments in Sweden. Scand J Gastroenterol 2012.

10. Pezzilli R, Uomo G, Gabbrielli A, et al. A prospective multicentre survey on the treatment of acute pancreatitis in Italy. Dig Liver Dis 2007;39:838-846.

11. Besselink MG, van Santvoort HC, Buskens E, et al. Probiotic prophylaxis in predicted severe acute pancreatitis: A randomised, double-blind, placebo-controlled trial. Lancet 2008;371:651-659.

12. Paye F, Rotman N, Radier C, et al. Percutaneous aspiration for bacteriological studies in patients with necrotizing pancreatitis. Br J Surg 1998;85:755-759.

13. Vincent JL, Rello J, Marshall J, et al. International study of the prevalence and outcomes of infection in intensive care units. JAMA 2009;302:2323-2329.
14. Kochhar R, Noor MT, Wig J. Fungal infections in severe acute pancreatitis. J Gastroenterol Hepatol 2011;26:952959.

15. Behrman SW, Bahr MH, Dickson PV, Zarzaur BL. The microbiology of secondary and postoperative pancreatic infections: Implications for antimicrobial management. Arch Surg 2011;146:613-619.

16. Kochhar R, Ahammed SK, Chakrabarti A, et al. Prevalence and outcome of fungal infection in patients with severe acute pancreatitis. J Gastroenterol Hepatol 2009;24:743-747.

17. Isenmann $\mathrm{R}$, Schwarz M, Rau B, et al. Characteristics of infection with Candida species in patients with necrotizing pancreatitis. World J Surg 2002;26:372-376.

18. Grewe M, Tsiotos GG, Luque de-Leon E, Sarr MG. Fungal infection in acute necrotizing pancreatitis. J Am Coll Surg 1999;188:408-414.

19. De Waele JJ, Vogelaers D, Blot S, Colardyn F. Fungal infections in patients with severe acute pancreatitis and the use of prophylactic therapy. Clin Infect Dis 2003;37:208213.

20. Wittau M, Mayer B, Scheele J, et al. Systematic review and meta-analysis of antibiotic prophylaxis in severe acute pancreatitis. Scand J Gastroenterol 2011;16:261-270.

21. Jafri NS, Mahid SS, Idstein SR, et al. Antibiotic prophylaxis is not protective in severe acute pancreatitis: A systematic review and meta-analysis. Am J Surg 2009;197:806-813.

Address correspondence to: Dr. Jan J. De Waele Department of Critical Care Medicine Ghent University Hospital De Pintelaan 185 9000 Gent, Belgium

E-mail: jan.dewaele@UGent.be 